\title{
Taenia crassiceps injection into the subarachnoid space of rats simulates radiological and morphological features of racemose neurocysticercosis
}

\author{
Pedro Tadao Hamamoto Filho ${ }^{1}$. Alexandre Todorovic Fabro ${ }^{2}$. \\ Marianna Vaz Rodrigues ${ }^{3}$. Rodrigo Bazan ${ }^{1}$ - Luiz Carlos Vulcano ${ }^{4}$. \\ Germano Francisco Biondi ${ }^{5}$ Marco Antônio Zanini ${ }^{1}$
}

Received: 1 June 2016 / Accepted: 31 August 2016/Published online: 9 September 2016

(C) Springer-Verlag Berlin Heidelberg 2016

\begin{abstract}
Purpose Neurocysticercosis is a major public health concern. Although its eradication appears feasible, the disease remains endemic in developing countries and has emerged again in Europe and in the USA. Basic studies on neurocysticercosis are needed to better understand the pathophysiologic mechanisms and, consequently, to improve treatment perspectives. Much has been published on experimental parenchymal neurocysticercosis, but there are no experimental models of racemose neurocysticercosis.

Methods Cysts of Taenia crassiceps were injected into the subarachnoid space of 11 rats. After 4 months, magnetic resonance imaging (MRI) was performed to verify the occurrence of ventricular dilatation and the distribution of cysts in the cerebrospinal fluid compartments. The histologic assessment was done focusing on changes in the ependyma, choroid plexus, and brain parenchyma.
\end{abstract}

Pedro Tadao Hamamoto Filho

pthamamotof@hotmail.com

1 Department of Neurology, Psychology and Psychiatry; Botucatu Medical School, UNESP - Univ. Estadual Paulista, Botucatu, SP, Brazil

2 Unit of Experimental Research; Botucatu Medical School, UNESP Univ. Estadual Paulista, Botucatu, SP, Brazil

3 Department of Microbiology and Immunology; Institute of Biosciences, UNESP - Univ. Estadual Paulista, Botucatu, SP, Brazil

4 Department of Animal Reproduction and Veterinary Radiology; School of Veterinary Medicine and Animal Science, UNESP - Univ. Estadual Paulista, Botucatu, SP, Brazil

5 Department of Veterinary Hygiene and Public Health; School of Veterinary Medicine and Animal Science, UNESP - Univ. Estadual Paulista, Botucatu, SP, Brazil
Results MRI and histologic assessment confirmed the findings similar to those seen in human racemose neurocysticercosis including enlargement of the basal cisterns, hydrocephalus, and inflammatory infiltration through the ependyma and choroid plexus into cerebrospinal fluid spaces.

Conclusions We developed a simple model of racemose neurocysticercosis by injecting cysts of $T$. crassiceps into the subarachnoid space of rats. This model can help understand the pathophysiologic mechanisms of the disease.

Keywords Neurocysticercosis · Racemose neurocysticercosis $\cdot$ Magnetic resonance imaging · Cerebrospinal fluid $\cdot$ Experimental model

\section{Introduction}

Neurocysticercosis is the most common parasitic disease of the central nervous system (CNS). It remains endemic in developing countries and migratory flows reintroduced the disease in Europe and in the United States [1, 2].

The clinical presentation of neurocysticercosis varies according to the number, location, size, and stage of development of the parasite within the CNS [3]. Cysts lodged in the subarachnoid space and ventricles elicit an important inflammatory response that can cause arachnoiditis, ependymitis, vasculitis, and cranial nerve entrapment. As a result, patients may develop hydrocephalus, stroke, neuropathies, and intracranial hypertension [4]. This is the racemose form of the disease, which is associated with its higher rates of incapacity, hospitalization, and death [5].

Studies on experimental neurocysticercosis have been published, but most of them focus on the host-parasite interaction within the brain parenchyma [6]. So, basic studies are needed to 
better understand the mechanisms underlying racemose neurocysticercosis [7]. Recently, we developed an experimental model of neurocysticercosis-induced hydrocephalus through the direct injection of cysts of Taenia crassiceps into the subarachnoid space of rats [8]. In this new study, we aimed to understand whether hydrocephalus is a consequence of mechanic obstruction of CSF flow or inflammatory reactions in this experimental model.

\section{Materials and methods}

The animals were handled according to ethical guidelines and current legislation. The board of the local Ethics Committee on Animal Use approved the project (CEUA 1038/2013).

Eleven adult Wistar rats (Rattus norvegicus) weighing 200 to $250 \mathrm{~g}$ were used. The animals were kept under adequate sanitary conditions in a temperature-controlled room $\left(21^{\circ} \mathrm{C}\right)$ under light-dark control. Food and water were available ad libitum.

\section{Parasites and inoculation}

The methods for parasite maintenance and inoculation were previously described [8]. Briefly, after aseptic removal from the peritoneal cavity of mice, 50 cysts were injected into the cisterns of each rat by suboccipital puncture with a $25 \mathrm{G}$ needle. All animals received general anesthesia by intraperitoneal injection of a mixture of ketamine $(100 \mathrm{mg} / \mathrm{ml})$ and xylazine $(20 \mathrm{mg} / \mathrm{ml})$ at a dose of $0.1 \mathrm{ml} / \mathrm{kg}$.

\section{Radiologic assessment}

Four months after inoculation, magnetic resonance imaging (MRI) using a $0.25 \mathrm{~T}$ equipment (Esaote, model Vet-MR)

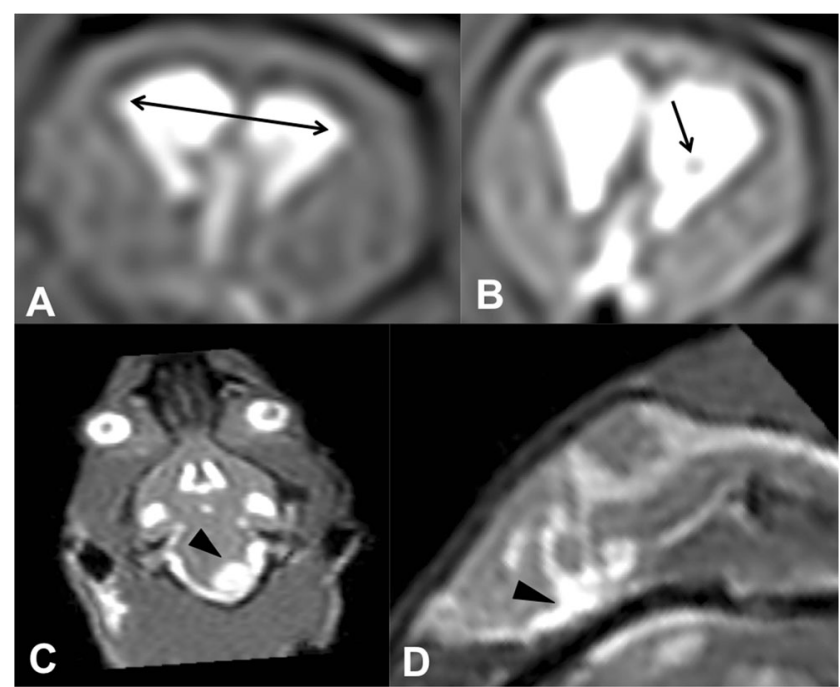

Fig. 1 T2-weighted gradient-echo MRI findings. a Note the ventricle enlargement. The double-arrow points to the maximal distance between frontal horns. b The arrow indicates a hyposignal inside the left lateral ventricle corresponding to a cyst. c, $\mathbf{d}$ Enlargement of the basal cisterns (arrowheads)

was performed to verify the occurrence of ventricular dilatation and distribution of cysts in the ventricles and basal cisterns. The protocol consisted of T2-weighted gradient-echo acquisitions $(0.6-\mathrm{mm}$ thickness; $\mathrm{T}$ eco $=5 \mathrm{~ms}$; $\mathrm{T}$ repetition $=10 \mathrm{~ms})$. Normal parameters of images from sham animals have been presented before [8].

\section{Histologic assessment}

Immediately after MRI, the animals were euthanized with an overdose of pentobarbital. The heart was perfused with buffered formalin and the encephala were removed, photographed, and fixed overnight in $10 \%$ neutral buffered formalin. Slices were cut at the level of the optic chiasm (to assess the lateral ventricle) and of the medulla
Table 1 Summary of imaging findings of the animals

\begin{tabular}{ccll}
\hline Animal & Bifrontal distance $(\mathrm{mm})$ & Compromised cisterns & Other findings \\
\hline 1 & 5.3 & None & - \\
2 & 7.9 & Cerebellar & - \\
3 & 9.2 & Cerebellar, supratentorial basal & - \\
4 & 7.6 & Cerebellar, brainstem & - \\
5 & 9.1 & Brainstem & - \\
6 & 9.5 & Cerebellar, supratentorial basal & Intraventricular cyst \\
7 & 6.1 & None & - \\
8 & 4.7 & Cerebellar & - \\
9 & 6.4 & Supratentorial basal & - \\
10 & 10.0 & Cerebellar & - \\
11 & 7.6 & Cerebellar, supratentorial basal & - \\
\hline
\end{tabular}




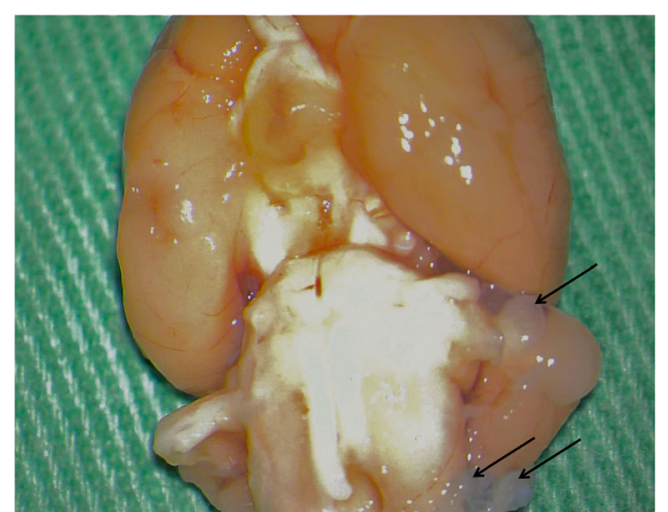

Fig. 2 Mesoscopic view showing cysts juxtaposed to the brainstem (arrows)

oblongata (to assess the fourth ventricle). Then, they were dehydrated in increasing alcohol concentrations, diafanized in xylene, and embedded in paraffin. The blocks were cut into $3-\mu \mathrm{m}$ sections and the sections were stained with hematoxylin-eosin.
Ventricle dilatation and changes in the ependymal epithelium, choroid plexus, and parenchyma were analyzed using stereologic methods [9].

\section{Results}

MRI showed ventricle enlargement in all animals. Nine animals exhibited enlargement of the basal cisterns. A cyst was detected inside the ventricle of one animal (Fig. 1). Table 1 summarizes the imaging findings of each animal. The mesoscopic view confirmed cysts juxtaposed to the brainstem (Fig. 2).

On histologic sections, cysts were observed inside all of the ventricles: lateral, third, and fourth ventricle (despite MRI detected only one large cyst within the lateral ventricle). Inflammatory cells were found in the choroid plexus of the fourth ventricle and in the ependyma. There was an increase in the cellularity of the brain
Fig. 3 A cyst can be seen inside the ventricle (a, arrow). Inflammatory cells are present in the ependyma (b, arrow) and in the choroid plexus (arrowhead). Note the lymphoplasmacytic infiltration in the choroid plexus (c, dotted line), which is thick and edematous (d). Astroglial proliferation can be seen in the periventricular region $(\mathbf{e}$, dotted line) and the ependymal epithelium is flattened (f), with loss of the normal cuboid pattern and cilia (dotted line)

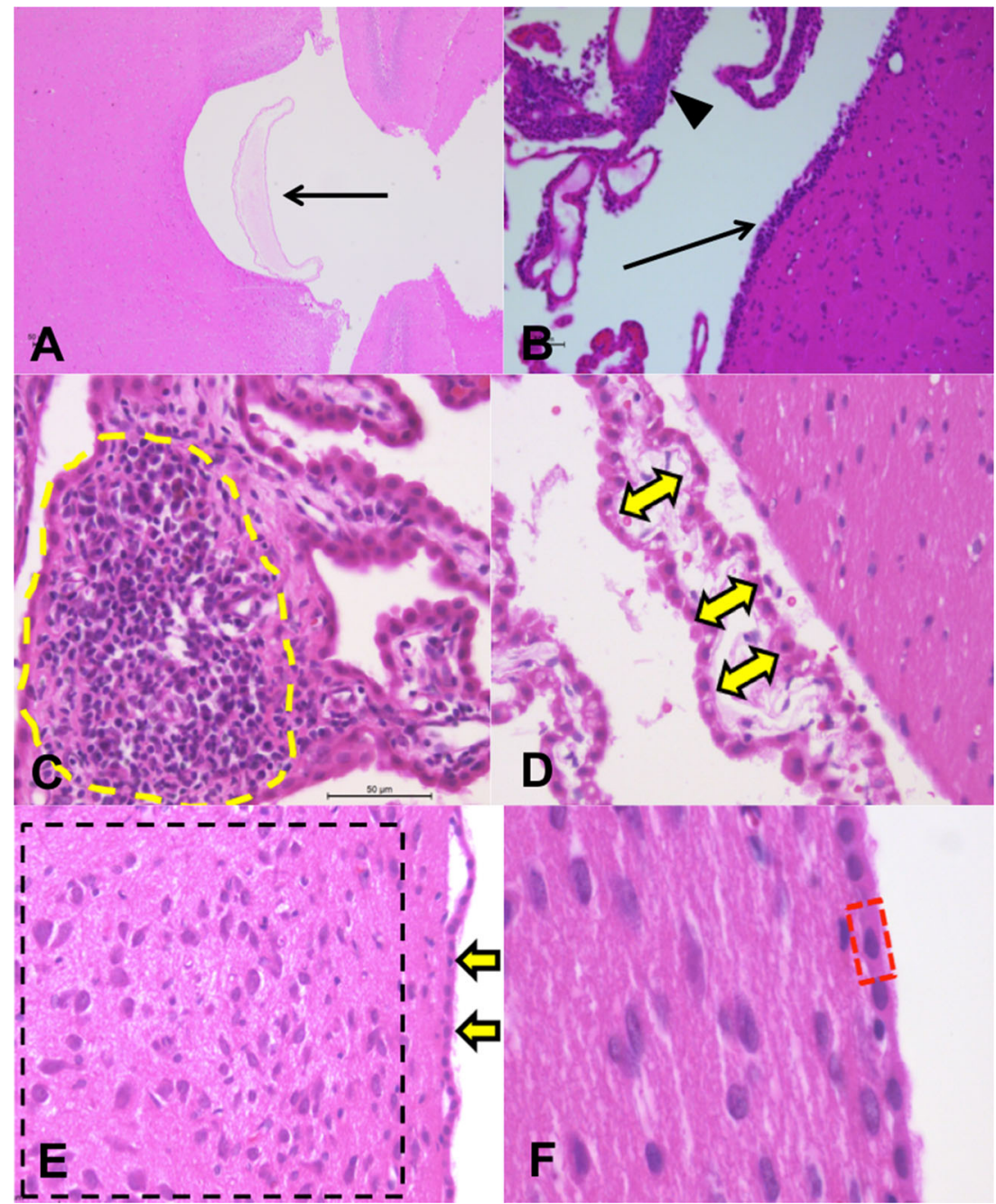


parenchyma near the ventricle cavity, which may correspond to reactive astrogliosis. The choroid plexus was thick and edematous. Finally, the ependyma was thin, flattened, and contained no cilia (Fig. 3).

\section{Discussion}

In human racemose neurocysticercosis, cysts in the CSF compartments (basal subarachnoid cisterns and ventricles) are typical findings and are useful for diagnostic purposes [10]. These cysts behave differently from those of the parenchymal form, i.e., membranes proliferate through the subarachnoid space and can reach a large size. The cysts can obstruct CSF flow and distort the adjacent brain parenchyma, causing different symptoms [11]. Degenerating cysts and the cysts themselves elicit inflammatory responses in the subarachnoid space, in the ventricle, and on the ependymal surface. These inflammatory processes can cause headache and vasculitis. The consequent scars in the subarachnoid space disturb the absorption and flow of CSF, leading to hydrocephalus [12].

Hydrocephalus occurs in up to $30 \%$ of patients with neurocysticercosis [13]. Despite advances in experimental studies of neurocysticercosis, ventricle enlargement has not been systematically found. Mattos-Silva et al. [14] reported asymmetrical ventriculomegaly as an anecdotal finding. In our previous pilot study, we achieved hydrocephalus by injecting cysts of Taenia crassiceps into the subarachnoid space of rats but we could not explain whether hydrocephalus was a mere consequence of mechanic obstruction for CSF free flow or whether inflammatory reactions played a role on the CSF flow disturbance [8]. Now, it becomes clear that inflammation has a role in the development of hydrocephalus. In this way, our model may be useful for the understanding of hydrocephalus in neurocysticercosis, which has a different evolution from other forms of hydrocephalus [14].

Ependymal disruption, choroid plexus edema, and reactive astrogliosis are common findings in experimental models of hydrocephalus [15]. In fact, the elevated pressure in the ventricles disrupts the ependyma. The CSF extravasates through the brain parenchyma in attempt to be absorbed by waterchannel molecules in the basement membrane of astrocytes feet to the vessels [16].

In our model, hydrocephalus could be the simple consequence of mechanical obstruction of the normal pathways for the CSF flow by the parasites. However, the identification of inflammatory infiltrates in the choroid plexus points to the occurrence of an inflammatory response in the CSF compartments. Inflammatory cells in the ependyma simulate the ependymitis that occurs in human neurocysticercosis. Thus, we experimentally reproduced inflammatory patterns of the disease. Additionally, dynamic and adaptive morphologic evidence indicates the establishment of a chronic physiopathologic process.
Teale and her group have extensively studied experimental parenchymal neurocysticercosis. The authors inoculated the cestode Mesocestoides corti through a burr hole into the parietal bone. Even though the cysts are not directly introduced into the brain parenchyma, the invasion capacity of this cestode permits it to quickly reach the parenchyma, causing an inflammatory process that was well documented by the authors [17-19]. Although these studies made important contributions to the understanding of neurocysticercosis, they do not explain all pathophysiologic changes that occur in the racemose form.

In fact, studies have attempted the intracranial injection of Taenia crassiceps, but the fact that this metacestode displaces the nervous tissue was seen as a disadvantage for a model of experimental neurocysticercosis [6]. On the other hand, displacement of CNS structures is a real concern in the racemose form of neurocysticercosis. In the model proposed here, we observed common findings of racemose neurocysticercosis.

In conclusion, racemose neurocysticercosis was simulated by simple subarachnoid injection of Taenia crassiceps, including cysts throughout the CSF compartments, hydrocephalus, basal cistern enlargement, ependymitis, and inflammatory infiltrates in the CSF space. This model could be useful for the understanding of racemose neurocysticercosis and opens new research perspectives.

Acknowledgments We thank Dr. Diego Generoso for helping with animal procedures. We thank Mr. Heraldo André Catalan Rosa for the support on magnetic resonance imaging acquisition.

\section{Compliance with ethical standards}

Conflict of interest The authors declare that there is no conflict of interest.

\section{References}

1. Flisser A, Sarti E, Lightowlers M, Schantz P (2013) Neurocysticercosis: regional status, epidemiology, impact and control measures in the Americas. Acta Trop 87:43-51. doi:10.1016/S0001-706X(03)00054-8

2. O'Neal SE, Flecker RH (2015) Hospitalization frequency and charges for neurocysticercosis, United States, 2003-2012. Emerg Infect Dis 21:969-976. doi:10.3201/eid2106.141324

3. Sotelo J (2011) Clinical manifestations, diagnosis, and treatment of neurocysticercosis. Curr Neurol Neurosci Rep 11:529-535. doi:10.1007/s11910-011-0226-7

4. Proaño JV, Torres-Corzo J, Rodrígues-Dalla Vecchia R, GuizarSahagun G, Rangel-Castilla L (2009) Intraventricular and subarachnoidal basal cisterns neurocysticercosis: a comparative study between traditional treatment versus neuroendoscopic surgery. Childs Nerv Syst 25:1467-1475. doi:10.1007/s00381-009-0933-4

5. Estañol B, Corona T, Abad P (1986) A prognostic classification of cerebral cysticercosis: therapeutic implications. J Neurol Neurosurg Psychiatry 49:1131-1134 
6. Alvarez JI, Mishra BB, Gundra UM, Mishra PK, Teale JM (2010) Mesocestoides corti intracranial infection as a murine model for neurocysticercosis. Parasitology 137:359-372. doi:10.1017 /S0031182009991971

7. Nash TE, Singh G, White AC, Rajshekhar V, Loeb JA, Proaño JV, Takayanagui OM, Gonzalez AE, Butman JA, DeGiorgio C, Del BOH, Delgado-Escueta A, Evans CA, Gilman RH, Martinez SM, Medina MT, Pretell EJ, Teale J, Garcia HH (2006) Treatment of neurocysticercosis: current status and future research needs. Neurology 67:1120-1127. doi:10.1212/01.wnl.0000238514.51747.3a

8. Hamamoto Filho PT, Zanini MA, Botta FP, Rodrigues MV, Bazan R, Vulcano LC, Biondi GF (2015) Development of an experimental model of neurocysticercosis-induced hydrocephalus. Pilot study. Acta Cir Bras 30:819-823. doi:10.1590/S0102-865020150120000005

9. Mouton PR (2014) Neurostereology: unbiased stereology of neural systems, first edn. Wiley-Blackwell, Oxford

10. Garcia HH (2003) Del Brutto OH (2003) Imaging findings in neurocysticercosis. Acta Trop 87:71-78. doi:10.1016/S0001-706 $\mathrm{X}(03) 00057-3$

11. Mahale RR, Mehta A, Rangasetty S (2015) Extraparenchymal (racemose) neurocysticercosis and its multitude manifestations: a comprehensive review. J Clin Neurol 11:203-211. doi:10.3988 /jen.2015.11.3.203

12. Sotelo J, Marin C (1987) Hydrocephalus secondary to cysticercotic arachnoiditis. A long-term follow-up review of 92 cases. J Neurosurg 66:686-689
13. Matushita H, Pinto FCG, Cardeal DD, Teixeira MJ (2011) Hydrocephalus in neurocysticercosis. Childs Nerv Syst 27:17091721. doi:10.1007/s00381-011-1500-3

14. Matos-Silva H, Reciputti BP, Paula EC, Oliveira AL, Moura VBL, Vinaud MC, Oliveira MAP, Lino-Júnior RS (2012) Experimental encephalitis caused by Taenia crassiceps cysticerci in mice. Arq Neuropsiquiatr 70:287-292. doi:10.1590/S0004-282X2012005000010

15. Del Bigio MR (1993) Neuropathological changes caused by hydrocephalus. Acta Neuropathol 85:573-585

16. Skjolding AD, Rowland IJ, Sogaard LV, Praetorius J, Penkowa M, Juhler M (2010) Hydrocephalus induces dynamics spatiotemporal regulation of aquaporin- 4 expression in the rat brain. Cerebrospinal Fluid Res 7:20. doi:10.1186/1743-8454-7-20

17. Cardona AE, Restrepo BI, Jaramillo JM, Teale JM (1999) Development of an animal model for neurocysticercosis: immune response is characterized by a predominance of $\gamma \delta \mathrm{T}$ cells. $\mathrm{J}$ Immunol 162:995-1002

18. Alvarez JI, Teale JM (2007) Differential changes in junctional complex proteins suggest the ependymal lining as the main source of leukocyte infiltration into ventricles in murine neurocysticercosis. $\mathrm{J}$ Neuroimmunol 187:102-113. doi:10.1016/j.jneuroim.2007.05.005

19. Alvarez JI, Teale JM (2008) Multiple expression of matrix metaloproteinases in murine neurocysticercosis: implications for leukocyte migration through multiple central nervous system barriers. Brain Res 1214:145-158. doi:10.1016/j.brainres.2008.03.036 